\title{
HomeCI - A visual editor for healthcare professionals in the design of home based care
}

\author{
Chris D Nugent, Member, IEEE, Richard J. Davies, Josef Hallberg, Mark P. Donnelly, Student \\ Member, IEEE, Kare Synnes, Michael Poland, Jonathan Wallace, Member, IEEE, Dewar Finlay, \\ Maurice Mulvenna and David Craig
}

\begin{abstract}
The demands of introducing a more practical means of managing and monitoring technology within the home environment to support independent living are increasing. Within this paper we present a prototype solution, referred to as HomeCI, which allows healthcare professionals to establish the conditions/rules within which technology in the home should operate. The HomeCI concept is based on the use of visual notation and has been designed for use by healthcare professionals with a non technical background. Within the paper we present the design of the first version of the HomeCI visual editor and present the results of a usability study conducted on 4 healthcare professionals.
\end{abstract}

\section{INTRODUCTION}

Advances in healthcare technologies which provide the ability of home based vital sign monitoring in conjunction with wearable healthcare systems and smart environments are beginning to revolutionise the way healthcare is being delivered and managed. This comes at a time when the general population have a genuine interest to take more control of their own healthcare management and indeed wish to have a deeper insight into its long term impact. This, coupled with demographic changes in the population and increases in the numbers of elderly in society [1], has provided not only an opportunity for the deployment of technology in home based healthcare, but also a real need for successful solutions to meet end user demands.

Many efforts have been directed towards the development of new and emerging technologies to further support the person in their own home for example fall detectors [2], behavioural analysis systems [3], intelligent garments [4, 5] to name but a few. Nevertheless, the real underlying need still exists in the development of an infrastructure which will support the delivery and management of the healthcare coupled with a realistic re-imbursement scheme. An additional requirement is the consideration which should be given to existing healthcare practice and how the introduction of new paradigms will impact upon this. This

Manuscript received April 16, 2007.

Chris D Nugent, School of Computing and Mathematics, University of Ulster, Belfast, Northern Ireland, (tel: +44 2890 368330, email: cd.nugent@ulster.ac.uk). Richard Davies, Mark Donnelly, Michael Poland, Johnny Wallace, Dewar Finlay and Maurice Mulvenna are with the School of Computing and Mathematics, University of Ulster, Belfast, Northern Ireland, (ri.davies@ulster.ac.uk, mp.donnelly@ulster.ac.uk, poland-m@ulster.ac.uk, jg.wallace@ulster.ac.uk, d.finlay@ulster.ac.uk, md.mulvenna@ulster.ac.uk). Josef Hallberg and Kåre Synnes are from Luleå University of Technology, Sweden, (josef.hallberg@ltu.se, kare.synnes@1tu.se). David Craig, Belfast City Hospital/Queen's University of Belfast, N. Ireland, (email: david.craig@qub.ac.uk) implicitly will require the support from both public and private healthcare providers to be flexible in the adoption of new solutions in addition to promoting their use.

In this paper we attempt to address the issue of how technology within the home environments can be managed by healthcare professionals. Specifically we attempt to address the problem of supporting healthcare professionals who wish to specify in a non-technical manner how the technology within the home should be monitored and what constitutes an abnormal situation. In the following Section we provide a more general overview to the Background to our study. In the Methods Section we describe the development of a visual editor, referred to as HomeCI, to support the design of home based healthcare rules in a non technical manner and in the Results Section we describe the evaluation of a prototype version of the HomeCI system. In the Discussions and Conclusions we discuss the initial impact of this concept following evaluation and explain how this work integrates with a larger study in the development of information management within smart environments.

\section{BACKGROUND}

Within the realms of home healthcare delivery terms such as service model or care model are commonly used as a means to represent the infrastructure through which the care is managed and delivered. Such an infrastructure can therefore be considered to act as a means of defining all of the stakeholders in the care provision process (including the patient) and how they interact with one another, in addition to defining and managing the devices and services and specifying how the care will actually be delivered [6, 7]. Although a vast amount of work has been focused towards the development of the home based technology, there is a deficit in terms of the amount of effort and indeed understanding which has been gained relating to the requirements of the healthcare operators of such systems. Within this paradigm healthcare operators may be from a number of professional backgrounds for example occupational therapy, physiotherapy, pharmacy, nursing and general medicine. All may have the responsibility to define the necessary requirements which will specify what type of technology should be included within the home environment and under which conditions should patterns of behaviour be considered as normal or abnormal. In addition to this, healthcare professionals may also be required to provide a form of intervention once instances of abnormal behaviour or alarm situations have actually been identified. 
The current study aims to address needs of healthcare professionals who manage the deployment of technology to support independent living. Specifically, our efforts have been directed towards the development of an interface for healthcare professionals to allow them to establish the conditions and rules which will govern and monitor technology to be deployed in a person's home. The approach is based on the design of a user interface which operates through the usage of visual notations. The premise of this approach aims to exploit the fact that the designer of the rules i.e. the healthcare professional will have limited knowledge of the backend processing of the monitoring system and will be largely from a non technical background.

\section{MethodS}

To undertake the proposed study required an assessment of typical situations which healthcare professionals would likely be faced with. In the second instance, it was required to develop a prototype tool (HomeCI) which would act as the interface between the healthcare provider and the care model. The following Sections elaborate upon these concepts in addition to providing the methodology adopted for the evaluation of the HomeCI prototype.

\section{A. Establishment of Scenarios}

To facilitate the design of a suitable interface a number of typical scenarios requiring home based support were established. Table 1 below summarises the five scenarios considered within the study.

TABLE I

SCENARIOS TO BE USED WITHIN STUDY TO ASSIST IN THE INITIAL DESIGN OF THE HOMECI USER INTERFACE TOOL

\begin{tabular}{|c|c|}
\hline Scenario & Summary \\
\hline Cooking & $\begin{array}{l}\text { The cooker should not be switched on until the person } \\
\text { has added some food to a pot and placed a pot on the } \\
\text { cooker. The cooker should not be switched on for more } \\
\text { than } 1 \text { hour. }\end{array}$ \\
\hline Dressing & $\begin{array}{l}\text { Once a person wakes and gets out of bed, within a } \\
\text { specified time ( } 30 \text { minutes) it is normal that they first go } \\
\text { to the bathroom and wash and then proceed to dress } \\
\text { themselves. Both of these activities should be performed } \\
\text { before the person leaves the house. }\end{array}$ \\
\hline Grooming & $\begin{array}{l}\text { Once in the bathroom the person should wash, brush } \\
\text { their teeth and comb their hair. There is no desired } \\
\text { sequence to these activities, however, the duration } \\
\text { within which they should be completed would not } \\
\text { normally exceed } 30 \text { minutes. }\end{array}$ \\
\hline $\begin{array}{l}\text { Medication } \\
\text { management }\end{array}$ & $\begin{array}{l}\text { A person must take their medication within } 1 \text { hour after } \\
\text { getting out of bed. Within this period they must also } \\
\text { ensure that they have had something to eat prior to taking } \\
\text { the medication. }\end{array}$ \\
\hline $\begin{array}{l}\text { Drink } \\
\text { Preparation }\end{array}$ & $\begin{array}{l}\text { The preparation of a drink should follow a general } \\
\text { sequence of steps. For example, the kettle should not be } \\
\text { boiled unless water has been added and the person should } \\
\text { not consider the activity finished until they have added } \\
\text { some milk or flavouring, tea/coffee and the boiled water } \\
\text { to a cup. }\end{array}$ \\
\hline
\end{tabular}

The scenarios were established in conjunction with a geriatric consultant, experienced in assessment of the requirements of patients living in their own homes in addition to having an appreciation of how technology could be deployed to support independent living [8]. All scenarios are based on the premise that the home environment will be highly sensorised hence the person's interaction with the environment and their movements can all be monitored.

\section{B. Development of HomeCI User Interface}

For the purposes of the prototype only the scenarios as in Table I have been considered. The HomeCI visual editor is based on the usage of visual notations. Figure 1 shows the initial constructs of the visual notation used.

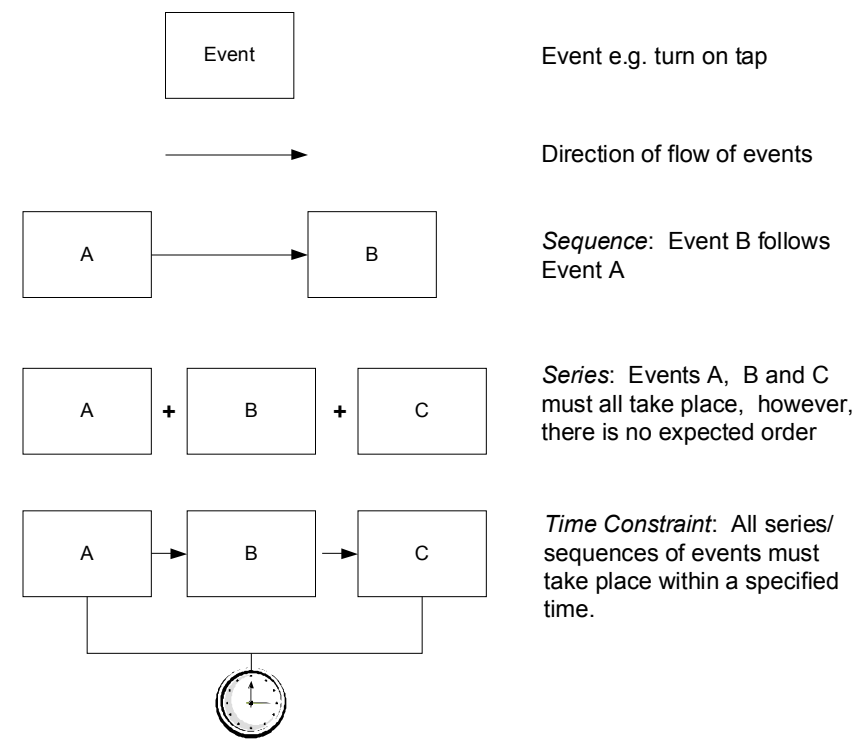

Figure 1 Graphical representation of visual notation within HomeCI user interface.

To avoid complexity in the initial version of the HomeCI prototype only sequences and series of events in addition to timing constraints of operations have been included. Increased complexity within the control structures will be addressed in the following version of the prototype.

The underlying computation of the system is therefore required to map the visual notation onto a form of computational logic which can then be converted into a computer language. Based on the control structures as presented in Figure 1 it has been possible to address this issue through the representation of the computational logic in terms of pseudo code. The pseudo code included in the example below provides an indication of how the HomeCI visual notation represented by the sequence event $A$ (last event) followed by event B (event) can be presented. In this example if event $B$ does not follow event $A$ an alarm is raised (ACTIVATE warning) otherwise the system proceeds without warning.

An example of the HomeCI visual editor developed within the study is shown in Figure 2. The visual editor was created using Macromedia Flash. This supported the development of a platform which could be easily deployed either on a mainstream operating system as a standalone application or alternatively deployed through a web browser. 


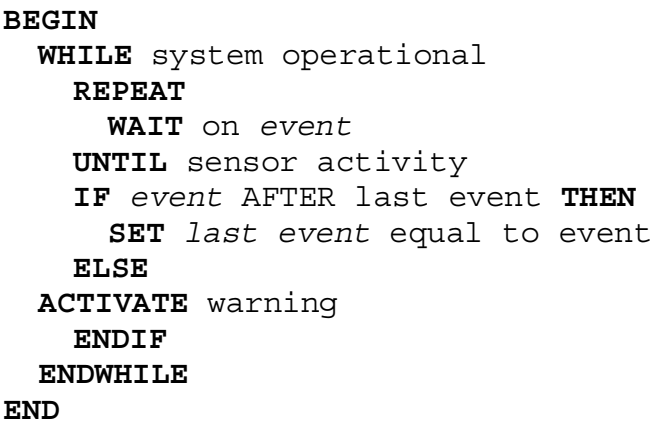

On the left hand side of the editor is a tool bar where users can select the events they wish to include in the design of the condition to be monitored. These events include for example 'turn on tap' or 'get out of bed'. All events are represented by icons which are representative of their meaning and in addition have a small textual description. Along the bottom of the editor are the necessary symbols to support the construction of the HomeCI notation (as presented in Figure 1). Both the events and the symbols can be dragged onto the main canvas of the application and can be freely moved around. For the purposes of evaluation a timer has been added to record the time taken to design one complete rule. The example presented in Figure 2 is representative of the 'Dressing' scenario as previously described in Table I.

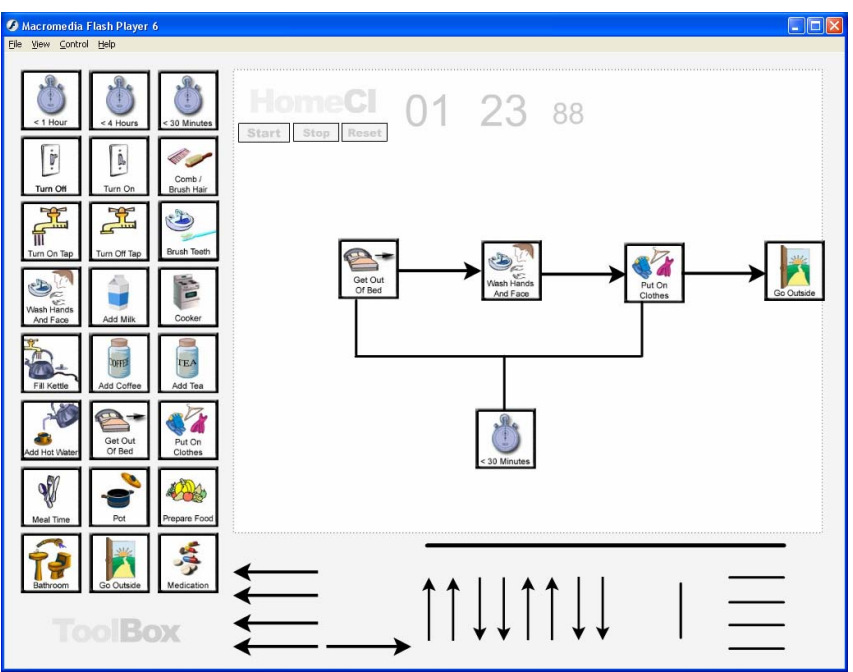

Figure 2. Screen Shot of HomeCI visual editor.

\section{Evaluation Approach for HomeCI Prototype}

To evaluate the HomeCI prototype a limited usability test with 4 users was designed. Two of the users were selected from Northern Ireland and two were selected from Northern Sweden. All users were experienced in working in the domain of elderly care and had good appreciation of the needs of technological solutions to be deployed in the home environment to support independent living. The professional backgrounds of the users were occupational therapy, registered nursing and physiotherapy. None of the users involved in the evaluation had ever written a computer program, nor were they familiar with general computer programming principles. All users were familiar with general usage of a PC and the Internet.

Prior to using the system the users were given a short presentation detailing the goals of the HomeCI concept which was followed by an explanation of the constructs used to build the rules with the visual notation. This was then followed by a brief demonstration of the HomeCI visual editor. This portion of the evaluation lasted for approximately 20 minutes. The evaluation was based on the scenarios as presented in Table 1 and structured as follows:

- Scenario 1 (Cooking): This example was shown to the users. The solution was constructed using HomeCI by the investigator who was conducting the evaluation.

- Scenario 2 (Dressing): Users were shown the final solution on the HomeCI editor and were shown how to revert back to the narrative from the visual notation.

- Scenario 3 (Grooming): Users were given the narrative and asked to create the rules using the HomeCI tool.

- Scenario 4 (Medication Management): Users were given the narrative and asked to create the rules using the HomeCI tool.

- Scenario 5 (Drink Preparation): Users were given the solution on the HomeCI editor and asked to create the narrative.

The users were not given any feedback or support during the evaluation. Upon completion of the evaluation the users where shown the desired results for Scenarios 3-5 and were then asked to complete a brief questionnaire.

\section{Results}

Table II presents the results from the evaluation for the 4 users involved. The user responses to Scenarios 3,4 and 5 were each assessed following the investigation. For Scenarios 3 and 4 each solution was assessed on 3 criteria; correct use of icons (maximum 3 marks), correct use of notation (maximum 2 marks) and correct insertion of timing constraints (maximum 2 marks). The time to complete both Scenarios 3 and 4 was also recorded. Overall this may be considered as a rudimentary assessment scheme, nevertheless, it can be considered sufficient to assess the current version of the system. (Assessment of more complex scenarios would require a more elaborate marking scheme.) For Scenario 5 the narrative produced was assessed and scored as a percentage. As can be seen from Table II the average percentage scores for all Scenarios were between $82.1 \%$ and $96.4 \%$. All users achieved either an equal or improved result in Scenario 4 in comparison to Scenario 3 and in addition a reduced amount of time required to complete the task was recorded in 3 out of the 4 users.

Following the evaluation the users were asked a number of questions regarding the HomeCI interface and asked to score their response between 1 and $10(1-$ poor, $10-$ excellent). The results from the users in terms of their experience of using the tool can be found in Table III. 
TABLE II

ASSESSMENT OF USER INTERACTION WITH HOMECI INTERFACE

\begin{tabular}{lccccc}
\hline \hline & $\mathbf{1}$ & $\mathbf{2}$ & $\mathbf{3}$ & $\mathbf{4}$ & Avg. \\
\hline Scenario 3 & 3 & 3 & 3 & 3 & 3 \\
Icons & 0 & 1 & 1 & 2 & 1 \\
Notation & 2 & 2 & 1 & 2 & 1.75 \\
Time & 71.4 & 85.7 & 71.4 & 100 & 82.1 \\
Total (\%) & 156 & 169 & 141 & 105 & 142.8 \\
Time (s) & & & & & \\
Scenario 4 & 3 & 3 & 3 & 3 & 3 \\
Icons & 2 & 2 & 2 & 2 & 2 \\
Notation & 2 & 1 & 2 & 2 & 1.75 \\
Time & 100 & 85.7 & 100 & 100 & 96.4 \\
Total (\%) & 87 & 100 & 113 & 119 & 104.7 \\
Time (s) & & & & & 86.2 \\
Scenario 5 & 75 & 95 & 85 & 90 & \\
Narrative (\%) & & & & & \\
\hline
\end{tabular}

TABLE III

USER'S EXPERIENCE IN THE EVALUATION OF THE HOMECI PROTOTYPE

\begin{tabular}{cccccc}
\hline \hline $\begin{array}{c}\text { Overall } \\
\text { experience }\end{array}$ & $\mathbf{1}$ & $\mathbf{2}$ & $\mathbf{3}$ & $\mathbf{4}$ & Avg. \\
\hline $\begin{array}{c}\text { Ease of using } \\
\text { icons }\end{array}$ & 8 & 8 & 7 & 8 & 7.75 \\
$\begin{array}{c}\text { Ease of use of } \\
\text { interface }\end{array}$ & 9 & 10 & 9 & 8 & 9 \\
$\begin{array}{c}\text { Ease to produce } \\
\text { narrative } \\
\text { Usefulness of } \\
\text { system }\end{array}$ & 8 & 9 & 5 & 9 & 7.75 \\
\hline
\end{tabular}

\section{DISCUSSION}

The design of the HomeCI editor has been based around the use of visual notations to avoid the technical complexities in establishing logic based rules. The HomeCI visual editor was evaluated by 4 healthcare professionals. Following a limited amount of training on this system all 4 users were able to construct two Scenarios and were also able to interpret and produce a narrative for a previously prepared solution. The performance of the users to develop the solutions ranged between $71.4 \%$ and $100 \%$. The main errors noted related to the incorrect usage of the notation. Following the evaluation users felt that they were not given enough time to learn these concepts, nevertheless, the performance achieved was still relatively high. In addition, the performance in producing a narrative based on a prepared solution had an average of $86.2 \%$. When the users were asked to evaluate their experience of using the tool all provided extremely positive feedback. Based on the series of questions asked (Table III) average scores of 7.75-9 out of 10 were achieved. All users reported a very high level of satisfaction in terms of being able to both use the system and understand the icons. In addition, all of the users felt that the general concept of HomeCI would be useful for home based care support. This feedback in a way validates the use of the HomeCI visual editor and has demonstrated how it is possible to use visual notation to allow those with a non- technical background to manage the deployment of technology within the home environment.

\section{CONCLUSIONS}

Within this study we have attempted to address the development of a user interface to support healthcare professionals in the design of managing technology which is to be deployed within a person's home. Such a tool has the potential to offer healthcare professionals a greater level of autonomy when using care management systems and reduce their reliance on technical support. Given the success of the initial usability study we intend to further develop the concepts of the HomeCI visual editor. In the first instance we intend to increase the functionality of the HomeCI notation in terms of the constructs it can represent. In conjunction with these proposed developments we intend to develop the underlying system which can parse the rules generated in the HomeCI prototype, and generate rules which can subsequently be easily deployed into a smart home. Consideration will also be directed towards the impact HomeCI will have on the daily work of the care providers and which organizational issues should be taken into account. Finally, the next stages of evaluation will include a larger number of users with a wider range of backgrounds.

\section{ACKNOWLEDGMENT}

The authors wish to express their thanks for the 4 users who evaluated the HomeCI prototype. The comments received following evaluation were extremely useful.

\section{REFERENCES}

[1] World Health Organisation, Ageing and Life course, http://www.who.int/ageing/en/ (accessed April 2007).

[2] G. Perolle, P. Fraisse, M. Mavros, I. Etxeberria, "Automatic fall detection and activity monitoring for elderly," Proceedings of MEDETEL, 2006.

[3] M. Pollack, "Intelligent technology for an aging population: the use of AI to assist elders with cognitive impairment," AI Magazine, vol. 26, no. 2, pp. 9-24, 2005.

[4] F. Axisa, P.M. Schmitt, C. Gehin, G. Delhomme, E. McAdams and A. Dittmar, "Flexible technologies and smart clothing for citizen medicine, home healthcare, and disease prevention," IEEE Transactions on Information Technology in Biomedicine, vol. 9, no. 3, 2005, pp. 325-336.

[5] C.D. Nugent, P.J. McCullagh, E.T. McAdams and A. Lymberis, Personalised Health Management Systems: The Integration of Innovative Sensing, Textile Information and Communication Technologies, IOS Press, Amsterdam, 2005.

[6] C.D. Nugent, M. Donnelly, R.K. Bali, A.N. Dwivedi, T. Quinn, R.N.G. Naguib, N.D. Black, "Towards a web-based knowledge management system for cardiovascular disease," Proceedings of EMBC2005, 2005.

[7] C. Paggetti, E Tamburini, "Remote management of integrated home care services: the DGHome platform," Proceedings of the $3^{\text {rd }}$ International Conference on Smart homes and health Telematics, ICOST2005, pp. 298-301, 2005.

[8] D. Craig, C. Nugent, M. Mulvenna, "Healthcare technologies for older people: what do physicians think?," Proceedings of the $4^{\text {th }}$ International Conference on Smart homes and health Telematics, ICOST2006, pp. 331-334, 2006. 